\title{
Anti-tumor enhancement of Fei-Liu-Ping ointment in combination with celecoxib via cyclooxygenase-2-mediated lung metastatic inflammatory microenvironment in Lewis lung carcinoma xenograft mouse model
}

\author{
Rui Liu ${ }^{1,2}$, Honggang Zheng ${ }^{1,2^{*}}$, Weidong Li ${ }^{1,2}$, Qiujun Guo ${ }^{1,2,3}$, Shulin He ${ }^{1,3}$, Yoshiro Hirasaki ${ }^{1,4}$, Wei Hou ${ }^{1,2}$, \\ Baojin Hua ${ }^{1,2^{*}}$, Conghuang Li ${ }^{1,2}$, Yanju Bao ${ }^{1,2}$, Yebo Gao ${ }^{1,2,3}$, Xin Qi ${ }^{1,2}$, Yingxia Pei ${ }^{1,2}$ and Yun Zhang ${ }^{1,2}$
}

\begin{abstract}
Background: Fei-Liu-Ping (FLP) ointment is an oral prescription medication that has been widely applied to treat lung cancer patients in China. Regulation of the metastatic microenvironment is an important therapeutic approach for prevention and treatment of tumor recurrence and metastasis. The advantage of Traditional Chinese Medicine management of lung cancer lies in the prevention of recurrence and metastasis. Our previous study has demonstrated that FLP ointment could regulate lung inflammatory microenvironment in vitro. However, the effects of FLP on the tumor microenvironment in vivo are still poorly understood. The objective of this study is to investigate the effect of FLP alone or in combination with celecoxib in the prevention of lung cancer progression by Cyclooxygenase (Cox)-2 mediated tumor inflammatory microenvironment in vivo.
\end{abstract}

Methods: 120 Lewis lung carcinoma xenograft mice were divided equally into four groups: vehicle, FLP, celecoxib, and FLP plus celecoxib. The dynamic growth of the xenografted tumors was observed using an in vivo fluorescence imaging system. Mice were sacrificed on day 14 , day 21 , and day 28 , and tumor specimens and lung tissues were harvested to detect the metastasis-associated protein expression.

Results: Tumor inhibition rate was $15.4,44.2,47.4 \%$ at day $14,37.3,34.7,61.5 \%$ at day 21 , and $15.5,10.3,32.5 \%$ at day 28 after treatment of FLP, celecoxib, and FLP plus celecoxib, respectively. Upon treatment of FLP and celecoxib together, lung metastasis rate was $30 \%$ (8 metastatic nodules) lower than other groups. FLP inhibited Cox-2 expression in a time-dependent manner. Moreover, FLP inhibited N-cadherin, matrix metalloproteinases (MMP)-9, and Vimentin expression. Treatment of FLP in combination with celecoxib was more effective than FLP or celecoxib alone in inhibiting vascular endothelial growth factor, platelet-derived growth factor receptors $\beta$, microsomal Prostaglandin E synthase-1, MMP-2, MMP-9, N-cadherin, and Vimentin expression, but increased E-cadherin expression.

Conclusions: FLP inhibited tumor growth and metastasis in a Lewis lung xenograft mice model through the Cox-2 pathway. FLP in combination with celecoxib enhanced the antitumor growth and anti-metastasis effects. Traditional Chinese herbs combined with anti-inflammatory drugs might offer a promising strategy to prevent tumor metastasis.

Keywords: Lung cancer, Fei-Liu-Ping ointment, Celecoxib, Inflammatory microenvironment

\footnotetext{
*Correspondence: macrogone@126.com; huabaojin@sohu.com

'Department of Oncology, Guang'anmen Hospital, China Academy of Chinese Medical Sciences, Number 5 Beixiange, Xicheng District, Beijing 100053, China

Full list of author information is available at the end of the article
} 


\section{Background}

Lung cancer is the leading cause of cancer-related deaths in the world [1]. Despite advances in surgical techniques, chemotherapy, and radiotherapy, the 5-year survival rate for lung cancer patients is lower than $20 \%$ [2]. The high mortality is likely attributed to early metastasis. Metastatic spread is the critical cause of mortality and treatment failure [3, 4]. Therefore, development of new therapies to treat patients with metastatic lung cancer is necessary to reduce mortality rates. Prevention of recurrence and metastasis is an important step towards management for lung cancer patients. Research on lung cancer has recently focused on tumor microenvironment and recognized that alterations in the tumor microenvironment are a predictor of tumor occurrence and metastasis [5]. Various agents targeting the tumor inflammatory microenvironment provide a new therapeutic method for preventing tumor metastasis [6]. Changes in the tumor microenvironment occur slowly, and has certain correlation with maintenance therapy to prevent tumor recurrence and metastasis. Currently, chemotherapeutic drugs and targeted agents are commonly used in maintenance therapy. Pemetrexed and Erlotinib are widely used as maintenance therapy for advanced non-small cell lung cancer [7, 8]; however, their adverse effects and high economic burden reduce patient compliance [9].

Tumor microenvironment plays a critical role in tumor growth, progression, and metastasis [10]. It is increasingly recognized that tumor inflammatory microenvironment is closely related to tumor metastasis [11-15]. Cyclooxygenase-2 (Cox-2) is a rate-limiting enzyme for prostanoid biosynthesis, and plays a crucial role in the tumor inflammatory microenvironment $[16,17]$. Cox- 2 is over-expressed in all the metastatic process of cancer and involved in angiogenesis, epithelial-mesenchymal transition (EMT) initiation, and extracellular matrix (ECM) destruction [18-21]. Increasing evidence has shown that the Cox-2 gene was constitutively over-expressed in most human solid tumors, such as colon cancer, breast cancer, and lung cancer, and could serve as a predictor of poor prognosis [22-24].

Inflammation is necessary to promote cancer initiation and progression through angiogenesis and change of tumor microenvironment [25]. Anti-inflammatory drugs have become indispensable for cancer prevention and treatment. Indeed, long-term use of non steroidal anti-inflammatory drugs (NSAIDs), such as aspirin, were shown to decrease the risk of colon cancer by 40-50\% [26]. Cox-2 inhibitors are amongst the most popular medications worldwide. The US Food and Drug Administration (FDA) approved celecoxib, which is a hallmark of COX-2 inhibitors used for tumor prevention, as the adjuvant therapy for treatment of familial adenomatous polyposis in 1999 [27].

Traditional Chinese Medicine (TCM), as an important part of complementary and alternative therapy, exhibit advantages in the prevention of tumor recurrence and metastasis compared with radiotherapy and chemotherapy [28]. TCM is often used for maintenance therapy of lung cancer mainly due to its low toxicity or adverse effects, less cost, and better improvement in clinical outcomes $[29,30]$. TCM scholars deem that Chinese herbs change the tumor microenvironment, such that tumor cells become dormant or difficult to survive in the "soil". The adverse effects or costs of Chinese herbs and antiinflammatory drugs are far lower than the targeted drugs or chemotherapy drugs. However, no previous experimental study has investigated the effect of Chinese herbs in combination with anti-inflammatory drugs for preventing tumor metastasis.

FLP ointment inhibits lung cancer invasion by regulating tumor inflammatory microenvironment through the NF- $\mathrm{kB}$ signaling pathway [31]. Celecoxib improves tumor microenvironment through inhibiting the Cox-2 pathway. Although FLP ointment has shown to be effective in treating lung cancer in vivo, the mechanisms of action remain unclear. Whether FLP ointment in combination with celecoxib exerts synergistic effects in improvement of tumor inflammatory microenvironment or prevents progression and metastasis is still poorly understood. The objective of the current study is to investigate the effect of FLP alone or in combination with celecoxib on tumor growth in Lewis lung xenograft model and Cox-2 expression in lung cancer specimens. Furthermore, we also sought to examine the effect of FLP on Cox-2, and other metastasis-related protein expression, in normal lung tissues.

\section{Methods}

\section{Drugs and antibodies}

FLP ointment (No. Z20063236) was prepared by Pharmaceutical preparation center of Guang'anmen Hospital, China Academy of Chinese Medical Sciences. Celecoxib (No. H20120355) was purchased from Pfizer Pharmaceuticals LLC (Dalian, China). Antibodies against Cox2, E-Cadherin, Vimentin, platelet-derived growth factor receptors (PDGFR) $\beta, \beta$-actin, GAPDH, anti-rabbit IgG, and horseradish peroxidase (HRP)-linked antibody was purchased from Cell Signaling Technology (Cambridge, MA, USA). Vascular endothelial growth factor (VEGF), N-Cadherin, microsomal Prostaglandin E synthase-1 (mPGES-1), matrix metalloproteinases (MMP)-2, and MMP-9 antibodies were purchased from Abcam (Cambridge, MA, USA). Biotinylated secondary antibodies were purchased from Beijing Xiya Jinqiao Biological Technology Co. Ltd. 


\section{Preparation of FLP ointment}

The herbs used in FLP ointment are the roots of Astragalus membranaceus (Fisch.) Bge.var.mongholicus (Bge.) Hsiao (Huang-Qi), Panax quinquefolium L. (XiYang-Shen), Ophiopogon japonicas (Thunb.) Ker-Gawl. (Mai-Dong), Glehnia littoralis Fr. Schmidt ex Miq. (BeiSha-Shen), Agrimonia pilosa Ledeb. (Xian-He-Cao), Polygonum bistorta L. (Quan-Shen), Patrinia villosa (Thunb.) Juss. (Bai-Jiang-Cao), Panax notoginseng (Burk.) F.H. Chen (San-Qi), Fritillaria cirrhosa D.Don (ChuanBei-Mu), Glycyrrhiza uralensis Fisch. (Gan-Cao), Cordycrps sinensis(Berk.) Sacc. (Dong-Cong-Xia-Cao), and the fruits of Prunus persica (L.) Batsch (Tao-Ren) and Prunus armeniaca L. var ansu Maxim. (Xing-Ren). All herbs were provided by the Guang'anmen Hospital and decocted twice with eightfold volume of distilled water for $1 \mathrm{~h}$. The decoction were collected, filtered, merged and concentrated to $2 \mathrm{~g} / \mathrm{mL}$ (equivalent to crude herb materials), and stored at $4{ }^{\circ} \mathrm{C}$ for oral use.

\section{Animals}

Specific pathogen free 6-8-weeks-old male C57BL/6 mice weighing $20 \pm 2$ g were purchased from Vital River Company (Beijing, China). All the mice were kept under a temperature and humidity-controlled animal facility with a 12-h light/dark cycle. Mice were had free access to feed pellets and tap water. All the experiments were carried out in accordance with the guidelines for animal experiments of China Academy of Chinese Medical Sciences (IACU numbers: SYXK [Beijing], 2012-0034).

\section{Cell culture}

LL/2-luc-M38 cells were kindly provided by the cancer institute of Guang'anmen Hospital, Chinese Academy of Science. Tumor cells were cultured in Dulbecco's modification of Eagle's medium Dulbecco (DMEM) medium containing $10 \%$ fetal bovine serum, $100 \mathrm{U} / \mathrm{ml}$ penicillin, and $100 \mathrm{mg} / \mathrm{ml}$ streptomycin in a cell culture incubator at $37{ }^{\circ} \mathrm{C}$ under $5 \% \mathrm{CO}_{2}$. Cells were collected at the logarithmic phase of growth by treatment with $0.25 \%$ trypsin for $1 \mathrm{~min}$, then the cell concentration was adjusted to $1 \times 10^{6}$ with phosphate buffer saline (PBS). Trypan Blue exclusion test indicated the number of living cells was greater than $95 \%$.

\section{Induction of Lewis lung cancer and intervention}

After 3-day acclimation, a subcutaneous injection of LL/2-luc-M38 cells $5 \times 10^{5}$ suspended in $0.2 \mathrm{~mL}$ PBS was implanted into the right flank of each $\mathrm{C} 57 \mathrm{BL} / 6$ mouse. Starting the next day, forty mice were randomly divided equally into four groups: the vehicle control group (received the normal saline by gavage), FLP ointment treated group (received $12 \mathrm{~g} / \mathrm{kg} /$ day FLP by gavage), celecoxib treated group (received celecoxib at doses of $3200 \mathrm{ppm} /$ day in the diet from date of implant until end of study [32]), and FLP ointment plus celecoxib group (received a combination of celecoxib and FLP treatments described above). The mice were treated up to 28 days, and some of the mice were sacrificed by cervical decapitation under ether anesthesia at day 14, day 21, and day 28 , and tumor specimens and normal lung tissues were harvested and weighed. Part of specimens were exposed to $4 \%$ paraformaldehyde fixation for hematoxylin-eosin (HE) staining and immunohistochemistry study, and the remaining tissues were kept in $-80{ }^{\circ} \mathrm{C}$ for Western blot analysis.

\section{In vivo bioluminescent imaging}

In order to observe the dynamic change of inhibitory effect of FLP on tumor growth, we compared in vivo tumor imaging with the volume of tumor from the sacrificed mice. Imaging was performed at day 7, 14, 21, and 28 after treatment ( $\mathrm{N}=6$ for each group). D-luciferin (Biotium, Hayward, CA, USA) was dissolved in $15 \mathrm{~g} / \mathrm{L}$ PBS and injected intraperitoneally at a dose of $10 \mu \mathrm{L} / \mathrm{g}$ body weight before imaging. Imaging was performed 40 min later, and then the mice were anesthetized under gas-induced anesthesia, and placed in the imaging chamber. Bioluminescent images were acquired by a cryogenically cooled charge-coupled device camera (IVIS Lumina Imaging System, Caliper Life Sciences Inc). A region of interest (ROI) was drawn from each tumor location and the signal was calculated based on the number of photons from mouse body surface area (per $/ \mathrm{s} / \mathrm{cm}^{2} / \mathrm{sr}$ ). Red signal represents the high intensity, and purple signal represents the low intensity. Finally, the collected data were analyzed by statistical analysis.

\section{Tumor inhibition rate}

Mice were sacrificed by cervical dislocation at day 14 , day 21 , and day 28 , and then the tumor tissue were harvested and weighed. Tumor inhibition rate was calculated as follows: Tumor inhibition rate $=(1-$ average weight of tumors in treatment group/average weight of tumors in control group) $\times 100 \%$.

\section{Histological staining and immunohistochemistry}

Tumor specimens and normal lung tissues were harvested and immersed into $4 \%$ paraformaldehyde fixation for $20 \mathrm{~h}$. All the paraffin embedded samples were cut into $5 \mu \mathrm{m}$ sections for HE staining, according to standard protocols. For immunohistochemistry study, each section was dehydrated through a graded series of ethanol solutions. Antigen retrieval was performed by incubating the specimens with ethylene diamine tetraacetic acid (EDTA) in a microwave oven for $150 \mathrm{~s}$. After rinsing with PBS for 
3 times, these sections were treated with $3 \%$ hydrogen peroxide for $10 \mathrm{~min}$ to inactivate endogenous peroxides. After blocking with $1 \%$ goat serum in PBS for $10 \mathrm{~min}$ at room temperature, sections were incubated with primary anti-Cox-2 (1:200 dilution), anti- mPGES-1 (1:100 dilution), anti- E-Cadherin (1:300 dilution), anti-N-Cadherin (1:300 dilution), anti-Vimentin (1:500 dilution), antiVEGF (1:50), anti-PDGFR $\beta$ (1:50), anti-MMP-2 (1:50), or anti-MMP-9 (1:200) antibodies overnight at $4{ }^{\circ} \mathrm{C}$. Sections were then incubated with horseradish peroxidase (HRP)-conjugated secondary antibody for $20 \mathrm{~min}$ at $37^{\circ} \mathrm{C}$ followed by incubation with diaminobenidine (DAB) for $3 \mathrm{~min}$. The slides were stained with hematoxylin, differentiated with $1 \%$ hydrochloric acid alcohol, and stained blue with $1 \%$ ammonia water. Finally stained tissues were analyzed by light microscopy. Negative controls were incubated with PBS instead of primary antibodies.

\section{Western blot analysis}

Proteins were isolated from tumor specimens and normal lung tissues and the expression levels were measured using Western blot analysis. Briefly, $100 \mathrm{mg}$ of the tumor specimens or normal lung tissues were minced on ice, washed 2 times with the cold PBS, and homogenized 20-40 times in $1 \mathrm{~mL}$ mixture of radio-immunoprecipitation assay (RIPA) buffer with protease inhibitor (Applygen Technologies Inc, Beijing, China). After an ice bath for $10 \mathrm{~min}$, homogenized samples were centrifuged at $1000 \mathrm{rpm}$ for another $10 \mathrm{~min}$, and the supernatants were collected. Protein concentration was calculated using standard bovine serum albumin (BSA) curve. Approximately $30 \mu \mathrm{g}$ of total proteins were separated by sodium dodecyl sulfate-polyacrylamide gel electrophoresis and electrophoretically transferred to nitrocellulose membranes. The membranes were blocked with $5 \%$ milk in Tris-buffered saline (TBS) for $2 \mathrm{~h}$ at room temperature, followed by incubation with anti-Cox-2 (1:1000 dilution), anti- mPGES-1 (1:500 dilution), anti-E-Cadherin (1:1000 dilution), anti-N-Cadherin (1:1000 dilution), anti-Vimentin (1:1000 dilution), anti-VEGF (1:500), antiPDGFR $\beta$ (1:1000), anti-MMP-2 (1:300), anti-MMP-9 (1:1000), anti- $\beta$-actin (1:1000), or anti-GAPDH (1:1000) antibodies. The membranes were incubated on a shaker overnight at $4{ }^{\circ} \mathrm{C}$ and then washed three times before incubating with the HRP-conjugated secondary antibodies at a dilution of 1:2000 for $1 \mathrm{~h}$ at room temperature. The bands were visualized by adding ECL detection reagent (Amersham Life Science, Piscataway, NJ, USA). The membranes were exposed to X-ray film and photographed by BIO-RAD ChemiDocXRS gel imaging system. The pictures were exported by using Quantity One software. The intensities of the protein bands for target protein were quantified relative to $\beta$-actin or GAPDH bands from the same sample using image analysis software ImageProPlus 4.5 (Diagnostic Instruments,USA).

\section{Statistical analysis}

All data are presented as mean \pm standard deviation (SD), while categorical variables are expressed as observed frequencies. One-way repeated-measures analysis of variance (ANOVA) and multiple comparisons tests were applied with Prism 6.0. All statistical tests were two-sided test, and $p$-values $<0.05$ were considered to be statistically significant.

\section{Results}

FLP ointment treatment exerted the best tumor growth inhibition rate at day 21 in Lewis lung cancer xenograft mice, and FLP in combination with celecoxib enhanced the antitumor effects.

Lewis lung cancer xenograft mice were used to evaluate the tumor growth inhibition effects of FLP, celecoxib, and FLP in combination with celecoxib. At five time points (day 0 , day 7 , day 14 , day 21 , and day 28 ) we performed in vivo bioluminescence imaging and collected tumor from mice sacrificed by cervical dislocation at 3 time points (day 14, day 21, and, day 28). The number of photons from mouse body surface area (Fig. 1a-c) and the tumor weight (Fig. 1d, e) were compared among each treatment group. In vivo bioluminescence imaging experiments showed that FLP ointment treatment significantly reduced the number of photons from the mouse body surface area $(p<0.001)$. Moreover, FLP ointment treatment significantly reduced tumor weight at day 21 compared with the control group $(p<0.01)$ and exhibited the highest tumor growth inhibition rate $(37.3 \%)$. These findings were consistent with in vivo imaging results. Specially, FLP ointment in combination with celecoxib was more effective in reducing per $/ \mathrm{s} / \mathrm{cm}^{2} / \mathrm{sr}$ and increasing the tumor growth inhibition rate compared with the FLP ointment alone, or celecoxib alone.

FLP ointment significantly inhibited the expression of Cox-2 in the tumor specimens at day 14, while in combination with celecoxib did not exert obvious advantages in inhibiting Cox-2 expression. Cox-2 is one of the key factors in maintaining the tumor inflammatory microenvironment. Cox-2 over-expression was found in lung cancer and was closely related to the prognosis of lung cancer patients [23]. We measured Cox-2 expression from the tumor specimens and normal lung tissue by immunohistochemistry and Western blot analysis at day 14 , day 21 , and day 28 . The results showed that there was no obvious change in Cox-2 expression in the control group over time. However, FLP, celecoxib, or FLP in 


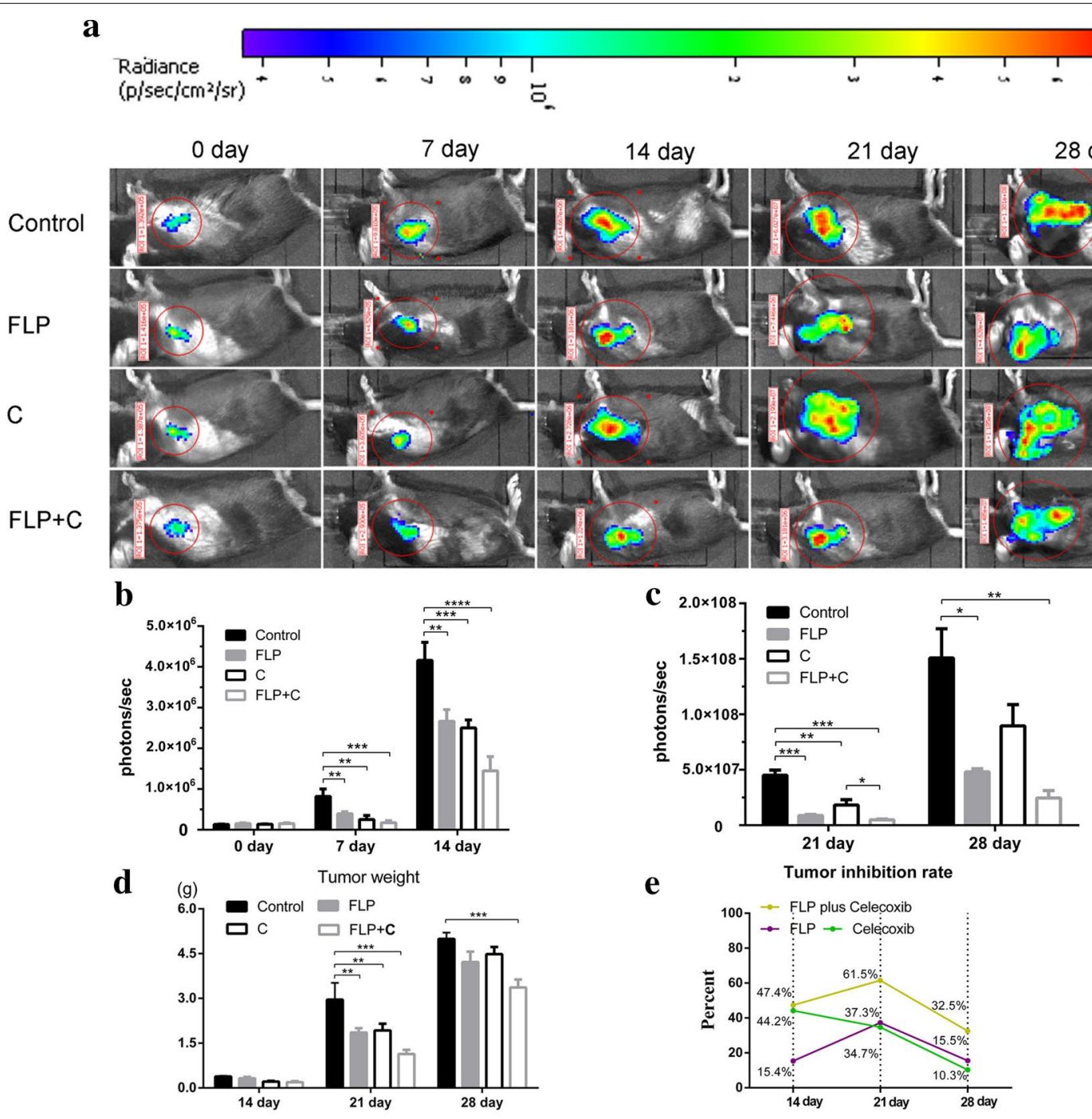

Fig. 1 Effects of FLP ointment on tumor growth in Lewis lung carcinoma xenograft mice. $5 \times 10^{5} \mathrm{LL} / 2-$ luc-M38 cells were implanted subcutaneously in C57BL/6 mouse. a Dynamic change of tumor growth measured by vivo bioluminescent signal; $\mathbf{b}$ The magnitude of tumor growth in vivo bioluminescent signal $\left(\mathrm{p} / \mathrm{s} / \mathrm{cm}^{2} / \mathrm{s}\right)$ at day 0 , day 7 , and day 14 . c The magnitude of tumor growth in vivo bioluminescent signal $\left(\mathrm{p} / \mathrm{s} / \mathrm{cm}^{2} / \mathrm{s}\right)$ at day 21 and day 28. d Tumor weight of the sacrificed mice at day 14, day 21, and day 28. e Tumor inhibition rate was calculated by $(1-$ average weight of tumors in treatment group/average weight of tumors in control group) $\times 100 \%$. Values were expressed as mean \pm SD. ${ }^{* *} p<0.01,{ }^{*} p<0.05$, ${ }^{* * *} p<0.001$, and ${ }^{* * *} p<0.0001$. FLP Fei-Liu-Ping ointment group, C celecoxib group, FLP + C Fei-Liu-Ping ointment plus celecoxib group. FLP ointment inhibited the tumor growth, while in combination with celecoxib exerted more significant anti-tumor growth effect at day 21 and day 28 , and these findings were consistent with in vivo imaging results

combination with celecoxib treatment inhibited Cox-2 expression compared with the control group at day 14 (Fig. 2). Interestingly, there was no significant difference in Cox-2 expression between celecoxib alone and FLP in combination with celecoxib at day 14, whereas only FLP in combination with celecoxib treatment significantly inhibited Cox-2 expression $(p<0.01)$ at day 21 compared with the control group. On day 28, there was no significant difference in Cox-2 expression between treatment groups and the control group.

\section{Large number of inflammatory cell infiltrations were} observed in the alveolar septum of Lewis lung cancer mice before and after metastasis

At day 28, we sacrificed mice and collected lung tissues to observe the rate of lung metastasis. The lung metastasis rate was $80,50,60$, and $30 \%$ and the total numbers of metastatic nodules were 52, 20, 29, and 8 in the control group, FLP group, celecoxib group, and FLP with celecoxib group, respectively. In order to examine the inflammatory changes in lung tissues, we observed 


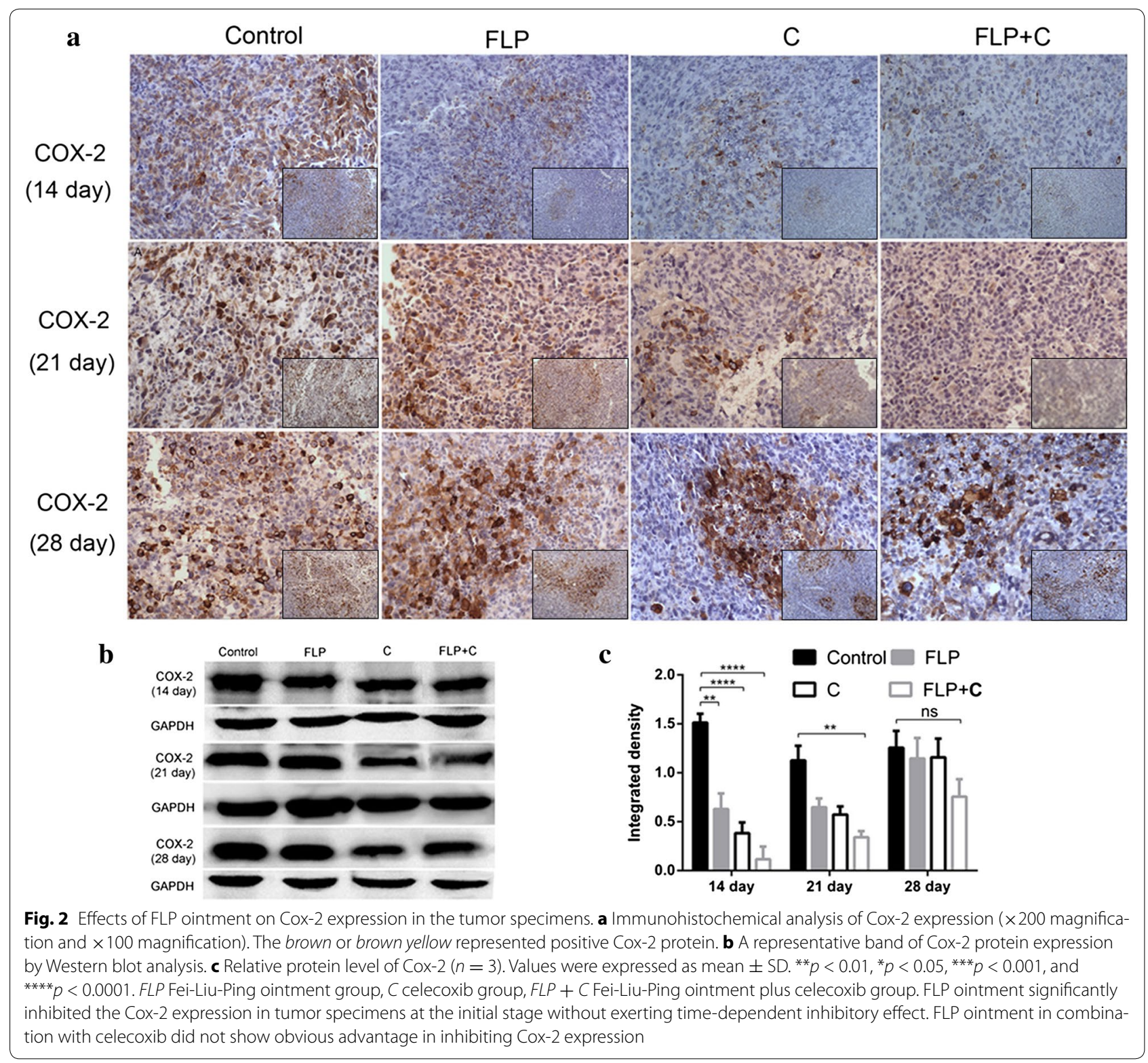

the changes of lung tissues before and after metastasis in the control group. The histological images indicated that a large number of inflammatory cell infiltrations were observed in the alveolar septum before the metastasis formation (Fig. 3a-day 14), and after the formation of metastases (Fig. 3a-day 21). With the progression of tumor metastases, large numbers of new inflammatory cell infiltrations in the alveolar septum of the location around the metastases were found (Fig. 3a-day 28). This has raised questions as follows: does the Cox-2 expression change along with the time sequence in the lung metastatic microenvironment? Can anti-tumor metastasis effect of FLP ointment, celecoxib, or FLP ointment in combination with celecoxib regulate tumor metastasis inflammatory microenvironment by inhibiting Cox- 2 expression? To better understand these questions, we conducted the following experiments.

FLP ointment in combination with celecoxib synergistically inhibited Cox-2 expression in the tumor metastasis microenvironment in a time-dependent manner

Cox-2 expression is known to contribute to tumor metastasis. We measured Cox-2 protein in the lung tissues by immunohistochemistry and Western blot analysis at day 14 , day 21, and day 28. The results showed that Cox-2 expression was increased in a time-dependent manner 


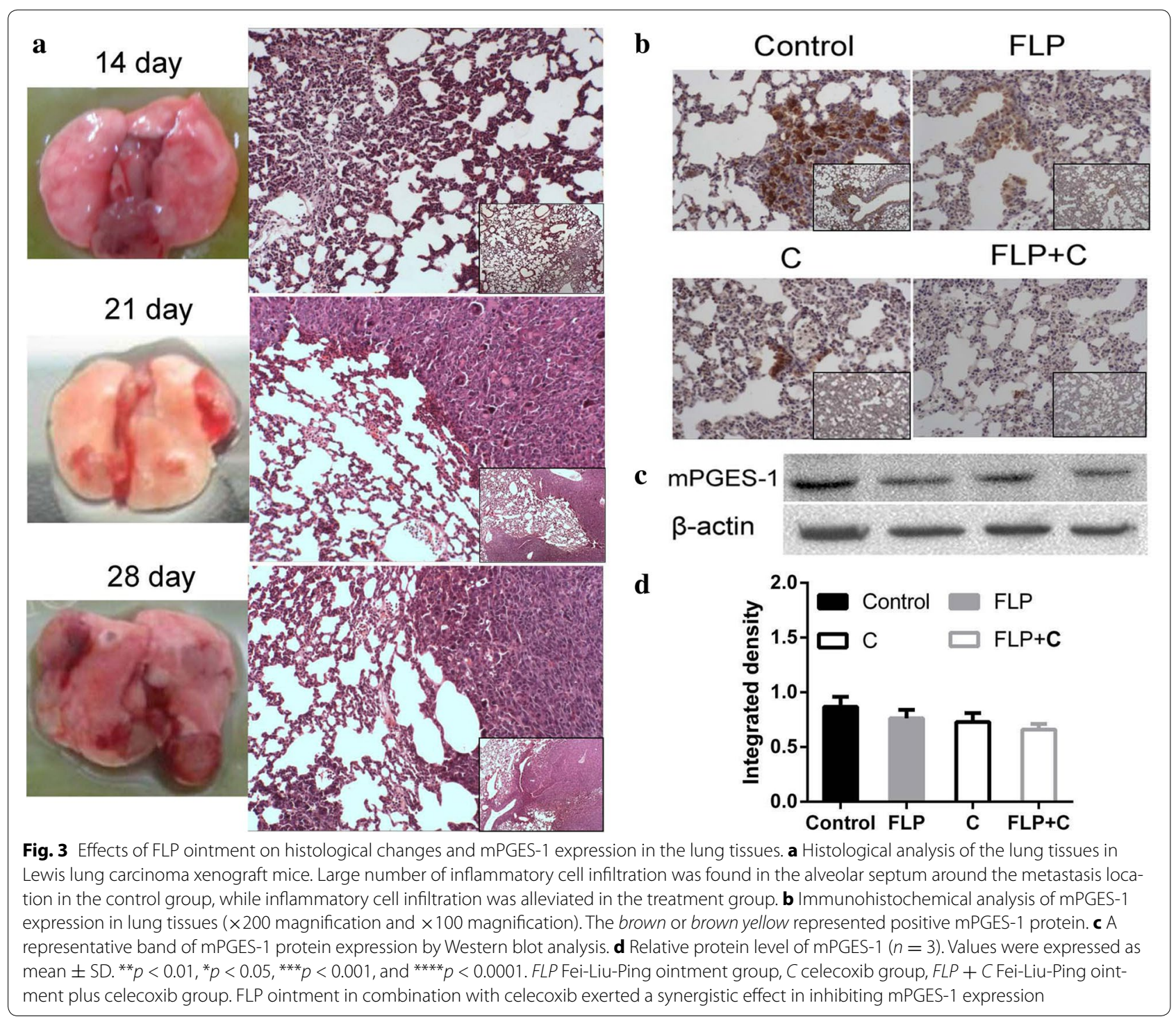

in the control group. FLP ointment markedly inhibited Cox-2 expression at day 21 compared with the control group $(p<0.05)$, and the inhibitory effect was increased at day $28(p<0.01)$. Celecoxib significantly inhibited Cox-2 expression at day 21 and day $28(p<0.01)$. FLP ointment in combination with celecoxib significantly inhibited Cox-2 expression over time, and the inhibitory effect was increased in a time-dependent manner (Fig. 4). In addition, inflammatory cell infiltration was attenuated to some extent in the treatment group. Since FLP ointment alone or FLP ointment in combination with celecoxib inhibited Cox-2 expression in a time-dependent manner, we determined that the strongest inhibition occurred at day 28 . We further investigated the changes of its downstream targets and metastasis related mPGES1, epithelial to mesenchymal transition (EMT), ECM, and angiogenesis-related protein expression.

\section{FLP ointment in combination with celecoxib reduced $\mathrm{mPGES}-1$ expression in the lung metastasis microenvironment}

Prostaglandin E2 (PGE2) can promote angiogenesis and tumor metastasis, attenuate cell apoptosis, and inhibit tumor immunity in various tumors [33]. PGE2 is derived from the enzymatic release of arachidonic acid, which is metabolized by Cox and specific PGE syntheses. The mPGES-1, a subtype of PGE syntheses, is an inducible enzyme that functions downstream 


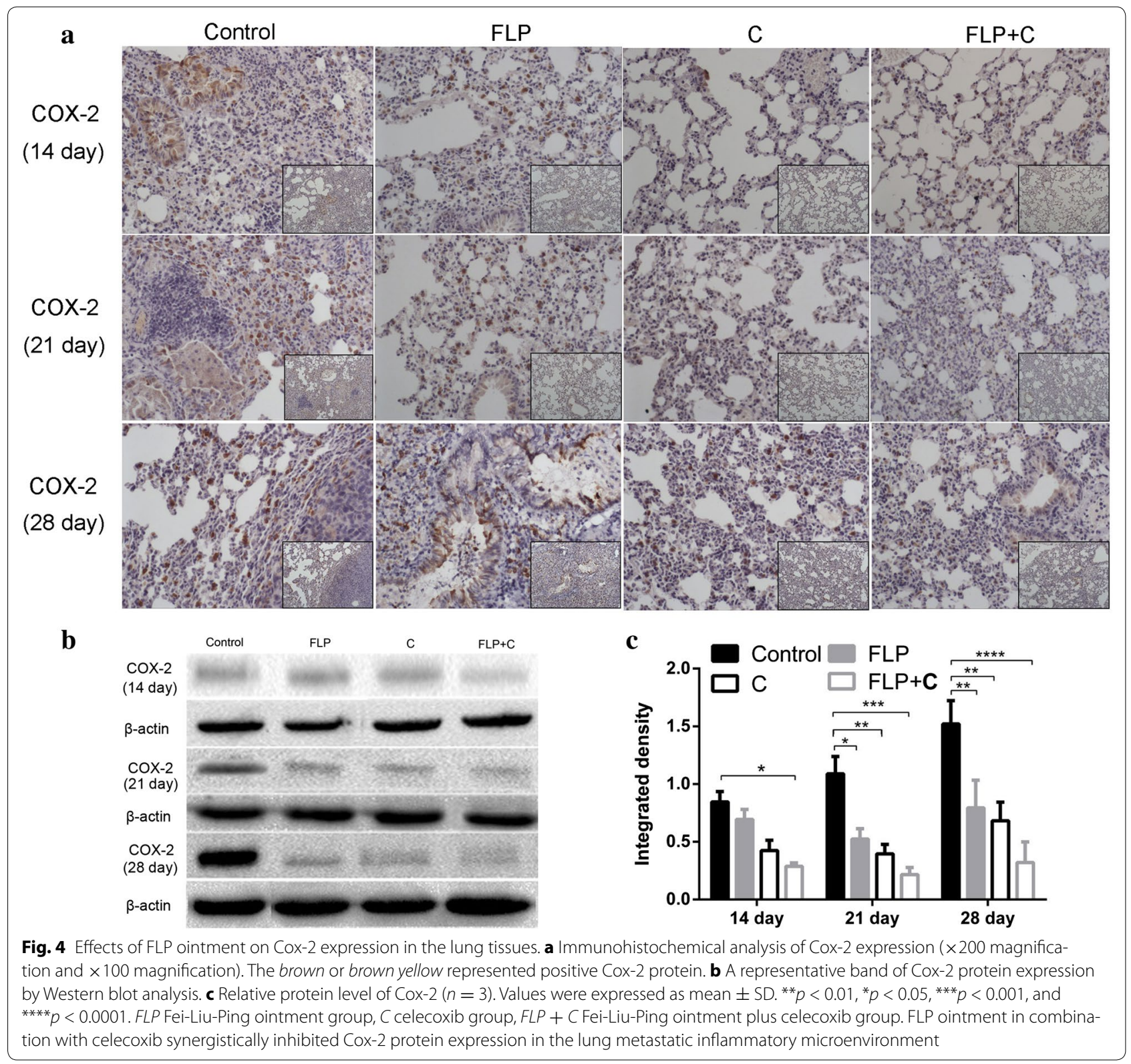

of Cox- 2 in the PGE2 -biosynthesis pathway and often over-expressed in non-small cell lung cancer [34]. Overexpression of mPGES-1 contributes to tumor growth, invasion, and metastasis [35]. As shown in Fig. 3b, mPGES-1 over-expression was identified in lung tissues of Lewis lung cancer mice. FLP ointment in combination with celecoxib was more effective than FLP ointment or celecoxib alone in inhibiting mPGES-1 expression. Immunohistochemistry image indicated that FLP ointment more effectively inhibited mPGES-1 expression than celecoxib alone, while Western blot results showed that there was no statistically significant difference in
mPGES-1 protein expression between the treatment and control groups $(p>0.05)$.

\section{FLP ointment in combination with celecoxib inhibited VEGF and PDGFR $\beta$ expression}

Angiogenesis is integral for tumor development, providing nutrients necessary for cell growth. Angiogenesis is required for the initiation of metastasis and plays an important role during initiation and end process of tumor metastasis. VEGF is the strongest angiogenesis stimulating factor [36]. PDGFR $\beta$ expression in endothelial cells regulates VEGF transcription and secretion. The 
PDGF/PDGF receptor pathway is located on pericytes of the tumor stroma, and plays a pivotal role in angiogenesis and vessel maturation [37, 38]. As shown in Fig. 5, FLP ointment significantly inhibited VEGF protein expression $(p<0.05)$. FLP ointment in combination with celecoxib exhibited the strongest inhibitory effect on VEGF protein expression $(p<0.001)$. Furthermore, FLP ointment combined with celecoxib significantly inhibited PDGFR $\beta$ protein expression $(p<0.01)$.

\section{FLP ointment in combination with celecoxib regulated EMT-related proteins expression in the lung tissues of Lewis lung cancer mice}

EMT is known to be involved in the invasion and metastasis of malignant tumor cells [39]. EMT is defined as switching of polarized epithelial cells to a migratory fibroblastoid phenotype. EMT refers the morphology from epithelial to mesenchymal transition and achieves the migratory capacity, which might be a bridge between inflammation and tumor [40, 41]. The characteristics of EMT include the loss of EMT-related proteins expression, such as E-cadherin and keratin, and the up-regulation of interstitial related proteins expression, including N-cadherin, Vimentin, and Fibronectin. We measured E-cadherin, N-cadherin, and Vimentin expression in the lung tissue of Lewis lung cancer mice by immunohistochemistry and Western blot analysis. As shown in Fig. 6, E-cadherin expression was reduced, and $\mathrm{N}$-cadherin and Vimentin expression was enhanced in the control group. FLP ointment increased E-cadherin expression, but significantly reduced $\mathrm{N}$-cadherin and Vimentin expression.

\section{FLP ointment in combination with celecoxib effectively reduced ECM-related proteins expression}

ECM is an important tissue barrier for tumor metastasis. Deregulation of the ECM and basement membrane is the basic prerequisite for the cancer cell invasion and angiogenesis [34, 42]. One of the critical factors for tumor invasion is a large production of MMPs that principally participates in the degradation of basement membranes and ECM [43]. Type-IV collagen is a major

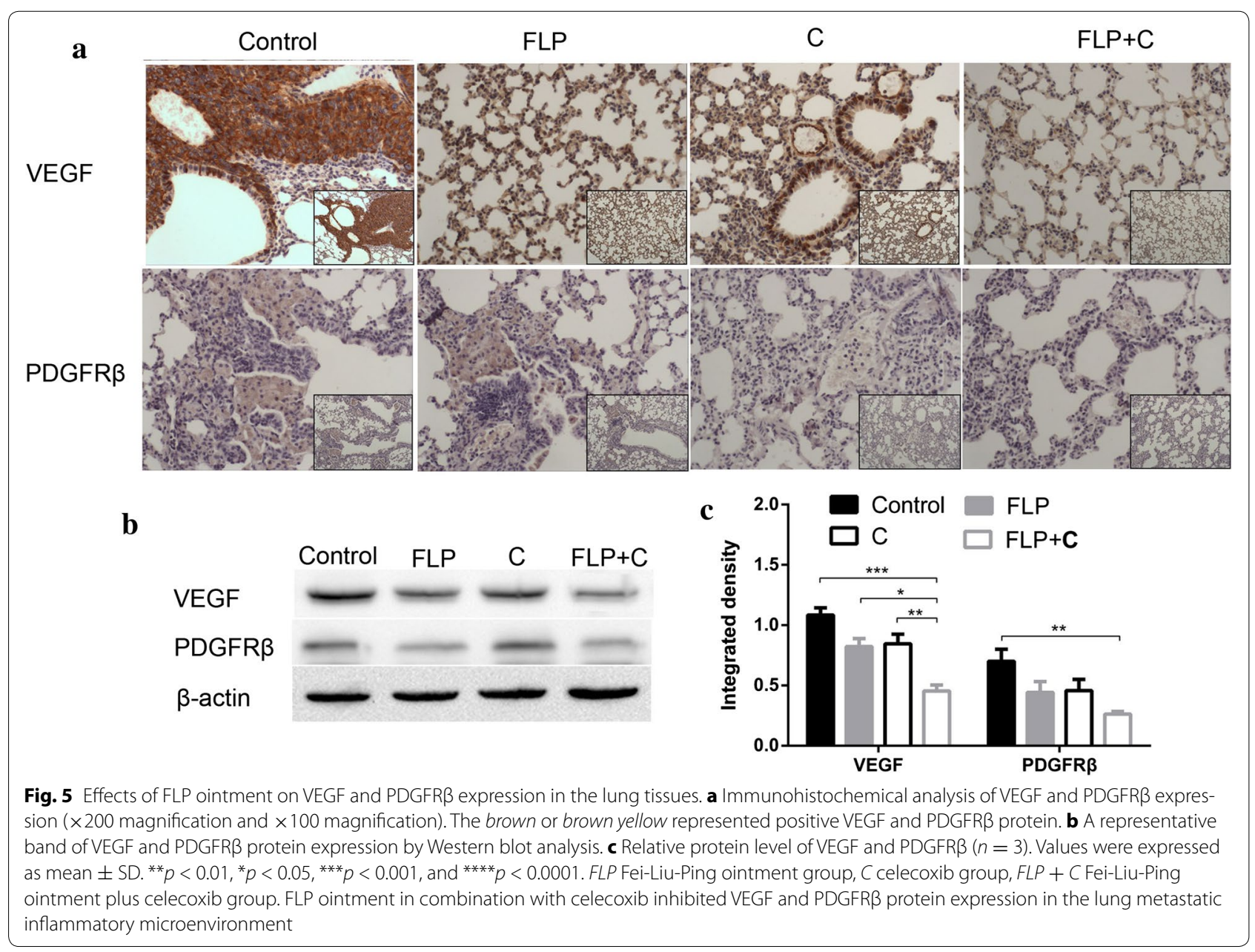




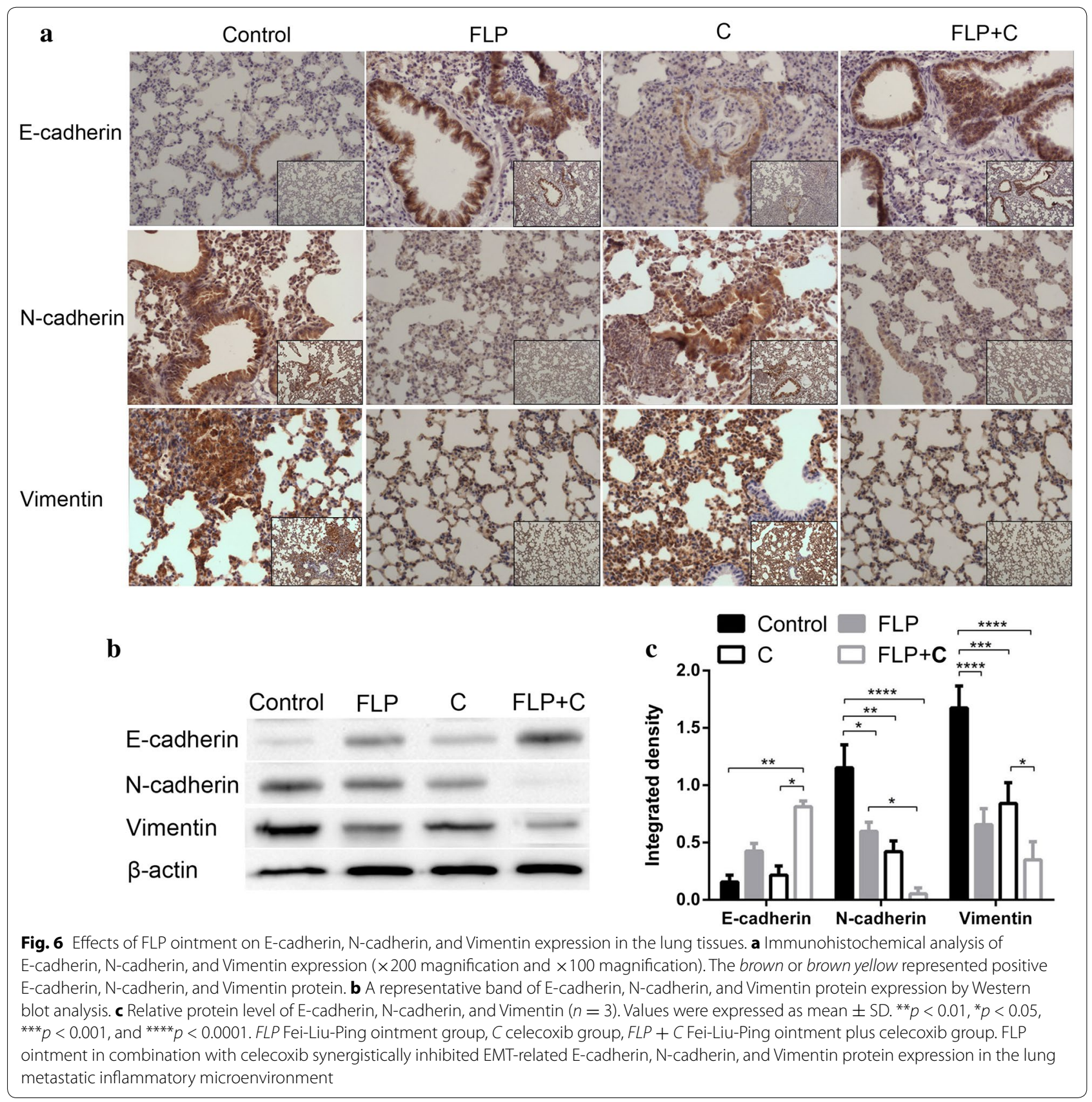

constituent of the basement membrane. The major MMPs involved in tumor angiogenesis are MMP-2 and MMP-9 [44]. MMP-2 and MMP-9 play critical roles in the degradation of type-IV collagen [45]. Moreover, MMP-9 mainly regulates the bioavailability of VEGF, the most potent inducer of tumor angiogenesis [46]. As shown in Fig. 7, FLP ointment inhibited the MMP-2 expression and MMP-9 $(p<0.001)$. FLP ointment in combination with celecoxib further inhibited the expression of MMP2 and MMP9.

\section{Discussion}

This study investigated the effect of FLP on the regulation of tumor metastatic inflammatory microenvironment. FLP ointment inhibited Cox-2 expression of the tumor specimens. More importantly, it also inhibited Cox-2 expression of the normal lung tissues in the tumor metastatic inflammatory microenvironment in a timedependent manner. Our study demonstrated for the first time that FLP ointment in combination with celecoxib adjunctly regulates Cox-2 mediated metastasis-related 


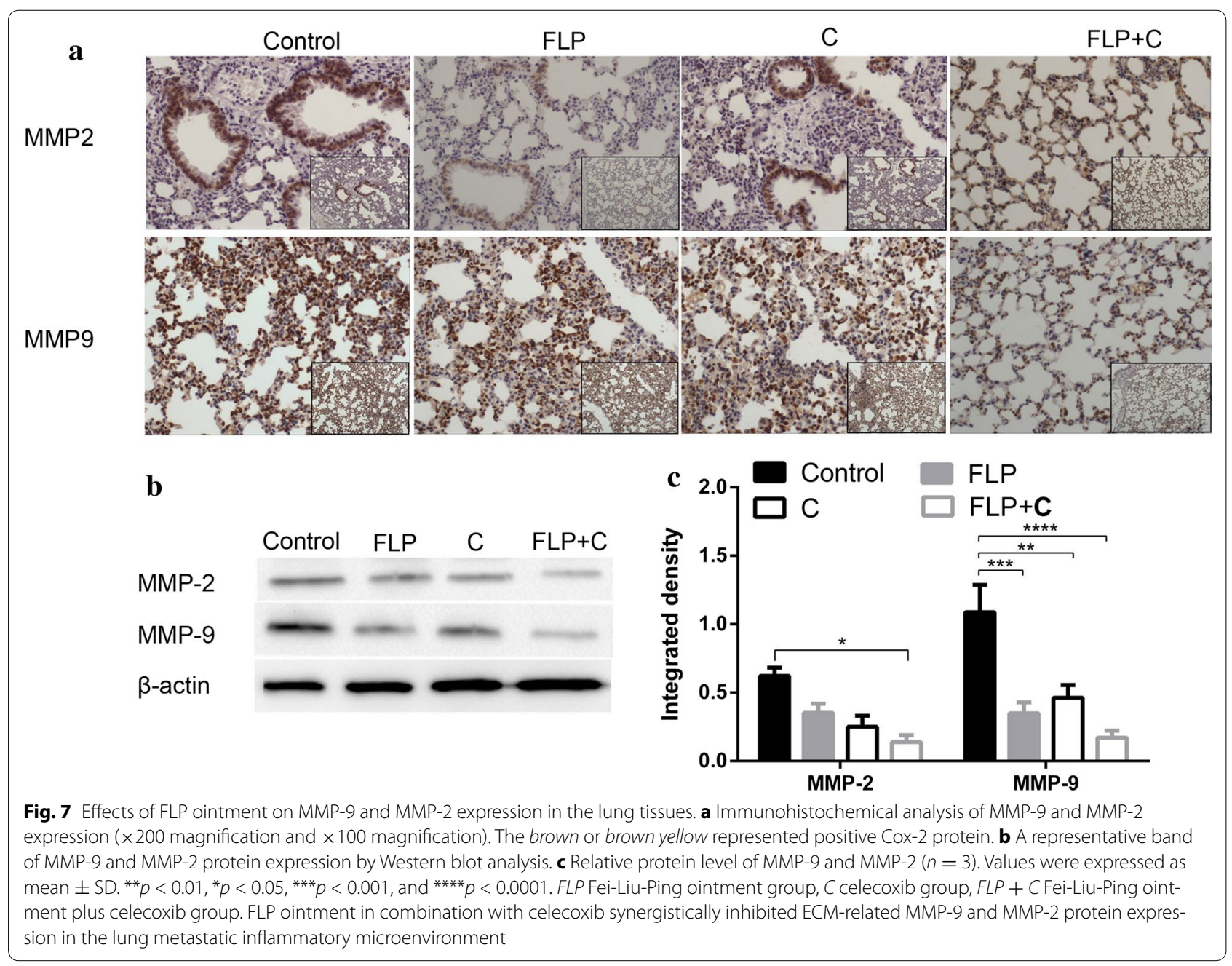

proteins expression in the microenvironment. Thus, integrated therapy provided better efficacy in the prevention of lung metastasis in the Lewis lung carcinoma xenograft mouse model.

FLP ointment has been used to treat lung cancer and has been suggested to prevent lung cancer metastasis or recurrence for many years in China [47]. FLP ointment has been developed based on the Differentiation of Syndrome of TCM theory for lung cancer or lung metastasis patients with deficiency of both Qi and Yin. FLP has potential to improve the quality of life, inhibit tumor growth, and prolong survival of lung cancer patients. Our previous study [31] showed that FLP promotes dendritic cell maturation and regulates tumor inflammatory microenvironment in vitro, and inhibits tumor growth and invasion through regulating NF- kappa B pathway in tumors of the Lewis lung carcinoma xenograft mouse model in vivo. Moreover, FLP also reduces serum TNF$\alpha$, IL- $1 \beta$, and IL- 6 level in a Lewis lung carcinoma xenograft mouse. The potential mechanism of action of FLP ointment on the lung cancer treatment and prevention is presented in Fig. 8. The current study indicated that the tumor inhibition rate was $37.3 \%$ at day 21 , and this finding was consistent with the absolute number of photons from mouse body surface area that was seen from in vivo bioluminescence imaging at day 21 . However, these results could not demonstrate the anti-tumor effect of FLP via inhibition of Cox-2 expression since FLP ointment only effectively inhibited Cox-2 expression at day 14. In addition, vivo bioluminescence imaging experiment might replace regularly sacrificed mice in the observation of the tumor changes.

Reducing tumor-associated inflammation and preventing recurrence and metastasis are crucial therapeutic strategies for the management of cancer patients. Chinese medicine practitioners believe that the biggest advantage of TCM is not only the inhibition of tumor growth, but also the prevention of tumor recurrence and metastasis. According to the basic theory of TCM, the concept of holism in TCM is consistent with the tumor 


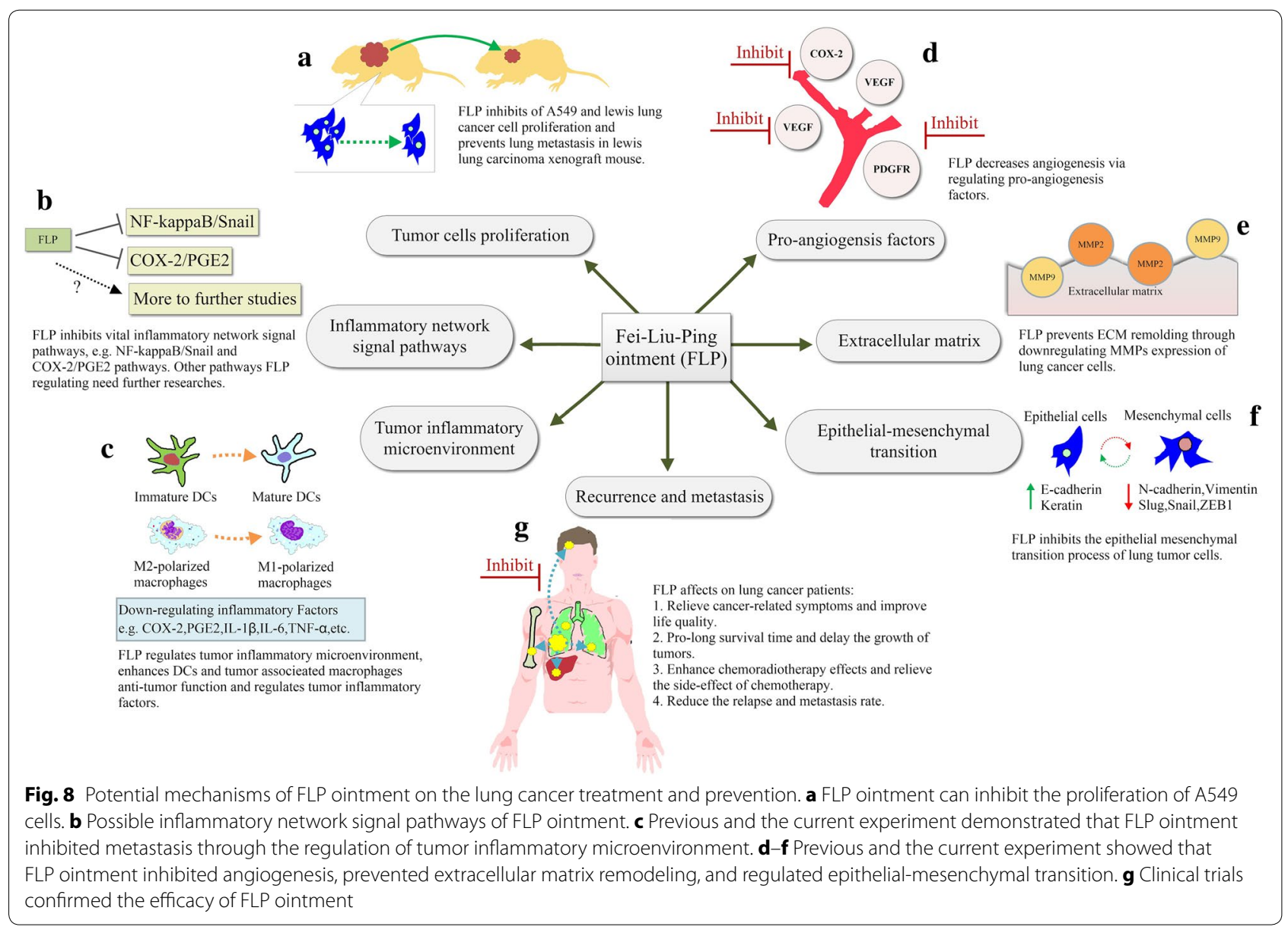

microenvironment just as macroscopical and microscopical aspects. Therefore, we hypothesized that the mechanism of TCM in preventing tumor recurrence and metastasis rely on regulation of the tumor microenvironment [31]. The crucial event in the course of lung cancer is its metastatic spread to distant organs. Metastatic cancer cells ('seeds') arise from the primary tumors need to create a specific organ microenvironments ('soil'), which is called organ targeting [48].The tumor microenvironment can be divided into three categories: core of primary tumor microenvironment, invasive tumor microenvironment, and metastatic tumor microenvironment [5]. We hypothesized that TCM therapies might prevent the affinity of "seed and soil" through regulating the tumor metastatic microenvironment and preventing tumor recurrence and metastasis. The current study also demonstrated increased Cox-2 expression in the lung metastatic microenvironment and that the inhibitory effect of FLP ointment on Cox-2 expression was strengthened over time. This suggested that TCM therapies reduce the risk of lung metastasis through effective regulation of the metastatic inflammatory microenvironment.

Previous studies have suggested that celecoxib inhibits Cox-2 expression in a variety of tumors, such as colon cancer, lung cancer, breast cancer, gastric cancer, and prostate cancer, and plays role in regulating tumor occurrence and progression [49-53]. However, the application of Cox-2 inhibitor for the treatment and prevention of cancer remains controversial. Anti-tumor effects of Cox-2 inhibitor alone or in combination with other chemotherapy drugs have been widely investigated, and many trials have shown inspiring results. For example, a Phase II study showed that treatment of celecoxib with cisplatin plus etoposide in extensive-stage small cell lung cancer was safe and feasible [54]. A phase I and pharmacokinetic studies demonstrated an antitumor effect of celecoxib in combination with ocetaxel and irinotecan [55]. The combination of the celecoxib and paclitaxel has been considered as the second-line therapy for non-small cell lung cancer [56]. On the contrary, chemotherapy or 
targeted drugs in combination with celecoxib did not improve the patient survival or remission rate in two clinical trials [57, 58]. Celecoxib inhibited tumor growth and lung metastasis in Lewis lung carcinoma xenograft C57BL/6 mice, and the mechanism was related to the early inhibition of the Cox-2 expression of the tumor specimens [49]. In our study, FLP ointment in combination with celecoxib exerted significant advantages in inhibiting Cox-2 expression in the lung metastatic microenvironment. The synergistic effect might be partly explained by the celecoxib-mediated stable and continuous inhibition of Cox-2 expression in lung metastatic microenvironment and FLP ointment- mediated inhibition of Cox-2 expression in a time-dependent manner (Fig. 4). FLP ointment inhibited Cox-2 and metastasisrelated downstream proteins. FLP ointment was superior to celecoxib in inhibiting MMP-9 and Vimentin protein expression. FLP might regulate these pathways via secondary inhibition of upstream pathways, or by directly inhibiting target protein expression. At the same, FLP ointment in combination with celecoxib synergistically inhibited angiogenesis and EMT or ECM- related protein expression.

To the best of our knowledge, no previous literature has reported the combined use of TCM and celecoxib in the treatment and prevention of cancer. Our findings could provide some basis for future clinical trials. Further studies are warranted to elucidate whether FLP ointment in combination with celecoxib can clinically provide the best efficacy in the prevention of recurrence and metastasis.

\section{Conclusions}

This study demonstrated that FLP ointment is effective in regulating the metastatic inflammatory microenvironment from multi-target via inhibition of Cox-2 expression and regulating lung metastasis-related proteins. Treatment of FLP ointment in combination with celecoxib adjunctly inhibits tumor growth and lung metastasis in a Lewis lung carcinoma xenograft mouse model. TCM combined with anti-inflammatory drugs might be a promising strategy in the prevention of tumor metastasis. Moreover, TCM might be a potential candidate drug of maintenance therapy. Further studies on anti-tumor and anti-metastatic effect of TCM may significantly contribute to the management of lung cancer patients.

\footnotetext{
Abbreviations

FLP: Fei-Liu-Ping; Cox: Cyclooxygenase; MMP: metalloproteinases; PDGFR: platelet-derived growth factor receptors; EMT: epithelial-mesenchymal transition; ECM: extracellular matrix; NSAIDs: non steroidal anti-inflammatory drugs; TCM: Traditional Chinese Medicine; VEGF: Vascular endothelial growth factor; ROI: region of interest; PGE2: Prostaglandin E2; mPGES-1: microsomal Prostaglandin E synthase-1.
}

\section{Authors' contributions}

Conceived and designed the experiments: BJH HGZ WDL RL Yoshiro Hirasaki. Performed the experiments: RL HGZ QJG LSH CHL YJB. Analyzed the data: RL WDL. Contributed reagents/materials/analysis tools: YBG XQ YXP YZ. Wrote the paper: RL HGZ. All authors read and approved the final manuscript.

\section{Author details}

${ }^{1}$ Department of Oncology, Guang'anmen Hospital, China Academy of Chinese Medical Sciences, Number 5 Beixiange, Xicheng District, Beijing 100053, China. ${ }^{2}$ Cancer Institute, China Academy of Chinese Medical Sciences, Beijing 100053, China. ${ }^{3}$ Beijing University of Chinese Medicine, Beijing 100029 China. ${ }^{4}$ Department of Japanese-Oriental (Kampo) Medicine, Graduate School of Medicine, Chiba University, 1-8-1 Inohana, Chuo-ku, Chiba 260-8670, Japan.

\section{Acknowledgements}

This study was supported by the grant from National Natural Science Foundation of China (No. 81273718, 81102719).

\section{Competing interests}

The authors declare that they have no competing interests.

Received: 24 October 2014 Accepted: 10 November 2015 Published online: 23 November 2015

\section{References}

1. Siegel R, Ma J, Zou Z, Jemal A. Cancer statistics, 2014. CA Cancer J Clin. 2014;64:9-29.

2. American Cancer Society: Cancer Facts and Figures 2013. 2013. http:// www.cancer.org/research/cancerfactsstatistics/cancerfactsfigures2013/.

3. Robert J. Biology of cancer metastasis. Bull Cancer. 2013;100:333-42.

4. Fidler IJ. The biology of cancer metastasis. Semin Cancer Biol. 2011;21:71.

5. Hanahan D, Weinberg RA. Hallmarks of cancer: the next generation. Cell. 2011;144:646-74

6. Finke JH, Rayman PA, Ko JS, Bradley JM, Gendler SJ, Cohen PA. Modification of the tumor microenvironment as a novel target of renal cell carcinoma therapeutics. Cancer J. 2013;19:353-64.

7. Greenhalgh J, McLeod C, Bagust A, Boland A, Fleeman N, Dundar Y, Oyee J, Dickson R, Davis H, Green J, et al. Pemetrexed for the maintenance treatment of locally advanced or metastatic non-small cell lung cancer. Health Technol Assess. 2010;14:33-9.

8. Dickson R, Bagust A, Boland A, Blundell M, Davis H, Dundar Y, Hockenhull J, Martin Saborido C, Oyee J, Ramani VS, . Erlotinib monotherapy for the maintenance treatment of non-small cell lung cancer after previous platinum-containing chemotherapy: a NICE single technology appraisal. Pharmacoeconomics. 2011;29:1051-62.

9. Jiang Y, Owonikoko TK, Ramalingam SS, Khuri FR, Belani CP. What is the role of maintenance therapy in the treatment of non-small cell lung cancer? Ther Adv Med Oncol. 2010;2:229-35.

10. Candido J, Hagemann T. Cancer-related inflammation. J Clin Immunol. 2013;33(Suppl 1):S79-84.

11. Spano D, Zollo M. Tumor microenvironment: a main actor in the metastasis process. Clin Exp Metastasis. 2012;29:381-95.

12. Jagannathan NR, Bhujwalla ZM. Tumor microenvironment in cancer treatment and metastasis. NMR Biomed. 2011;24:559-60.

13. Heinrich EL, Walser TC, Krysan K, Liclican EL, Grant JL, Rodriquez NL, Dubinett SM. The inflammatory tumor microenvironment, epithelial mesenchymal transition and lung carcinogenesis. Cancer Microenviron. 2012;5:5-18.

14. de Visser KE, Coussens LM. The inflammatory tumor microenvironment and its impact on cancer development. Contrib Microbiol. 2006;13:118-37

15. Mantovani A. Cancer: Inflaming metastasis. Nature. 2009;457:36-7.

16. Rizzo MT. Cyclooxygenase-2 in oncogenesis. Clin Chim Acta. 2011:412:671-87.

17. Greenhough A, Smartt HJ, Moore AE, Roberts HR, Williams AC, Paraskeva C, Kaidi A. The COX-2/PGE2 pathway: key roles in the hallmarks of cancer and adaptation to the tumour microenvironment. Carcinogenesis. 2009:30:377-86 
18. Li C, Pan T, Li J, Wei X, Chen T, Hu M, Wang Y. Study of COX-2 expression and angiogenesis in non-small cell lung cancer. Zhongguo Fei Ai Za Zhi. 2004; 7:501-4

19. Neil JR, Johnson KM, Nemenoff RA, Schiemann WP. Cox-2 inactivates Smad signaling and enhances EMT stimulated by TGF-beta through a PGE2-dependent mechanisms. Carcinogenesis. 2008;29:2227-35.

20. Liu H, Yang Y, Xiao J, LV Y, Liu Y, Yang H, Zhao L. COX-2-mediated regulation of VEGF-C in association with lymphangiogenesis and lymph node metastasis in lung cancer. Anat Rec (Hoboken). 2010;293:1838-46.

21. Shan Y, Zhang L, Bao Y, Li B, He C, Gao M, Feng X, Xu W, Zhang X, Wang S. Epithelial-mesenchymal transition, a novel target of sulforaphane via COX-2/MMP2, 9/Snail, ZEB1 and miR-200c/ZEB1 pathways in human bladder cancer cells. J Nutr Biochem. 2013;24:1062-9.

22. Stasinopoulos I, Shah T, Penet MF, Krishnamachary B, Bhujwalla ZM. COX-2 in cancer: Gordian knot or Achilles heel? Front Pharmacol. 2013;4:34.

23. Hida T. Overexpression of COX-2 and a potential clinical application of its inhibitors in lung cancer. Nihon Rinsho. 2004;62:1357-62.

24. Spugnini EP, Porrello A, Citro G, Baldi A. COX-2 overexpression in canine tumors: potential therapeutic targets in oncology. Histol Histopathol. 2005;20:1309-12.

25. Mantovani A, Allavena P, Sica A, Balkwill F. Cancer-related inflammation. Nature. 2008;454:436-44.

26. Giovannucci E, Egan KM, Hunter DJ, Stampfer MJ, Colditz GA, Willett WC, Speizer FE. Aspirin and the risk of colorectal cancer in women. N Engl J Med. 1995:333:609-14.

27. North GL. Celecoxib as adjunctive therapy for treatment of colorectal cancer. Ann Pharmacother. 2001;35:1638-43.

28. Tian X, Liu L. Effect and advantage of orally taking Chinese herbal medicine for treatment of lung cancer. Zhongguo Zhong Yao Za Zhi. 2010;35:2795-800.

29. Liu LS, Shen LP, Jiang Y, Han ZF, Hong J. Effect of integrated Chinese medical treatment (as maintenance therapy) on the survival time of patients with advanced non-small-cell lung cancer: a clinical study. Zhongguo Zhong Xi Yi Jie He Za Zhi. 2014;34:526-30.

30. Li SG, Chen HY, Ou-Yang CS, Wang XX, Yang ZJ, Tong Y, Cho WC. The efficacy of Chinese herbal medicine as an adjunctive therapy for advanced non-small cell lung cancer: a systematic review and meta-analysis. PLoS One. 2013;8:e57604

31. Li W, Chen C, Saud SM, Geng L, Zhang G, Liu R, Hua B. Fei-Liu-Ping ointment inhibits lung cancer growth and invasion by suppressing tumor inflammatory microenvironment. BMC Complement Altern Med. 2014;14:153.

32. Masferrer JL, Leahy KM, Koki AT, Zweifel BS, Settle SL, Woerner BM, Edwards DA, Flickinger AG, Moore RJ, Seibert K. Antiangiogenic and antitumor activities of cyclooxygenase-2 inhibitors. Cancer Res. 2000;60:1306-11.

33. Oshima $\mathrm{H}$, Oshima M. The inflammatory network in the gastrointestinal tumor microenvironment: lessons from mouse models. J Gastroenterol. 2012;47:97-106

34. Deryugina El, Quigley JP. Matrix metalloproteinases and tumor metastasis. Cancer Metastasis Rev. 2006;25:9-34

35. Nakanishi M, Gokhale V, Meuillet EJ, Rosenberg DW. mPGES-1 as a target for cancer suppression: A comprehensive invited review "Phospholipase A2 and lipid mediators". Biochimie. 2010;92:660-4.

36. Fushida S, Oyama K, Kinoshita J, Yagi Y, Okamoto K, Tajima H, Ninomiya I, Fujimura T, Ohta T. VEGF is a target molecule for peritoneal metastasis and malignant ascites in gastric cancer: prognostic significance of VEGF in ascites and efficacy of anti-VEGF monoclonal antibody. Onco Targets Ther. 2013:6:1445-51.

37. Fredriksson L, Li H, Eriksson U. The PDGF family: four gene products form five dimeric isoforms. Cytokine Growth Factor Rev. 2004;15:197-204.

38. Ostman A. PDGF receptors-mediators of autocrine tumor growth and regulators of tumor vasculature and stroma. Cytokine Growth Factor Rev. $2004 ; 15: 275-86$
39. Lee JM, Dedhar S, Kalluri R, Thompson EW. The epithelial-mesenchymal transition: new insights in signaling, development, and disease. J Cell Biol. 2006;172:973-81.

40. Lopez-Novoa JM, Nieto MA. Inflammation and EMT: an alliance towards organ fibrosis and cancer progression. EMBO Mol Med. 2009;1:303-14.

41. Zhou C, Liu J, Tang Y, Liang X. Inflammation linking EMT and cancer stem cells. Oral Oncol. 2012;48:1068-75.

42. Liotta LA, Tryggvason K, Garbisa S, Hart I, Foltz CM, Shafie S. Metastatic potential correlates with enzymatic degradation of basement membrane collagen. Nature. 1980;284:67-8.

43. Eccles SA, Welch DR. Metastasis: recent discoveries and novel treatment strategies. Lancet. 2007;369:1742-57.

44. Kessenbrock K, Plaks V, Werb Z. Matrix metalloproteinases: regulators of the tumor microenvironment. Cell. 2010;141:52-67.

45. Curran S, Murray Gl. Matrix metalloproteinases: molecular aspects of their roles in tumour invasion and metastasis. Eur J Cancer. 2000;36:1621-30.

46. Turpeenniemi-Hujanen T. Gelatinases (MMP-2 and -9) and their natural inhibitors as prognostic indicators in solid cancers. Biochimie. 2005;87:287-97

47. Piao BK, Tang WX, Zhang ZQ, Lin HS, Duan FW, Yu GQ. Clinical observation of Feiliuping ointment treatment for the advanced primary lung cancer: a clinical analysis of 339 cases. J Tradit Chin Med. 1991;32:21-3.

48. Fidler IJ. The pathogenesis of cancer metastasis: the 'seed and soil' hypothesis revisited. Nat Rev Cancer. 2003;3:453-8.

49. Yoshinaka R, Shibata MA, Morimoto J, Tanigawa N, Otsuki Y. COX-2 inhibitor celecoxib suppresses tumor growth and lung metastasis of a murine mammary cancer. Anticancer Res. 2006;26:4245-54.

50. Xin X, Majumder M, Girish GV, Mohindra V, Maruyama T, Lala PK. Targeting COX-2 and EP4 to control tumor growth, angiogenesis, lymphangiogenesis and metastasis to the lungs and lymph nodes in a breast cancer model. Lab Invest. 2012;92:1115-28.

51. Swamy MV, Herzog CR, Rao CV. Inhibition of COX-2 in colon cancer cell lines by celecoxib increases the nuclear localization of active p53. Cancer Res. 2003;63:5239-42.

52. Walther Z. COX-2 and angiogenesis in gastric cancer. J Clin Gastroenterol. 2003;37:4-6.

53. Katkoori VR, Manne K, Vital-Reyes VS, Rodriguez-Burford C, Shanmugam C, Sthanam M, Manne U, Chatla C, Abdulkadir SA, Grizzle WE. Selective COX-2 inhibitor (celecoxib) decreases cellular growth in prostate cancer cell lines independent of p53. Biotech Histochem. 2013;88:38-46.

54. Aruajo AM, Mendez JC, Coelho AL, Sousa B, Barata F, Figueiredo A, Amaro T, Azevedo I, Soares M. Phase II study of celecoxib with cisplatin plus etoposide in extensive-stage small cell lung cancer. Cancer Invest. 2009:27:391-6.

55. Argiris A, Kut V, Luong L, Avram MJ. Phase I and pharmacokinetic study of docetaxel, irinotecan, and celecoxib in patients with advanced non-small cell lung cancer. Invest New Drugs. 2006;24:203-12.

56. Gasparini G, Meo S, Comella G, Stani SC, Mariani L, Gamucci T, Avallone A, Lo Vullo S, Mansueto G, Bonginelli P, et al. The combination of the selective cyclooxygenase-2 inhibitor celecoxib with weekly paclitaxel is a safe and active second-line therapy for non-small cell lung cancer: a phase II study with biological correlates. Cancer J. 2005;11:209-16.

57. Agarwala A, Fisher W, Bruetman D, McClean J, Taber D, Titzer M, Juliar B, Yu M, Breen T, Einhorn LH, Hanna N. Gefitinib plus celecoxib in chemotherapy-naive patients with stage IIIB/IV non-small cell lung cancer: a phase II study from the Hoosier Oncology Group. J Thorac Oncol. 2008;3:374-9.

58. Mutter R, Lu B, Carbone DP, Csiki I, Moretti L, Johnson DH, Morrow JD, Sandler AB, Shyr Y, Ye F, Choy H. A phase II study of celecoxib in combination with paclitaxel, carboplatin, and radiotherapy for patients with inoperable stage IIIA/B non-small cell lung cancer. Clin Cancer Res. 2009;15:2158-65 